\title{
Multiple-RAT selection for reducing call blocking/ dropping probability in cooperative heterogeneous wireless networks
}

\author{
Olabisi E Falowo* and H Anthony Chan
}

\begin{abstract}
There is an increasing demand for high bandwidth-consuming services such as real-time video and video streaming over wireless access networks. A single radio access technology (RAT) in a heterogeneous wireless network may not always have enough radio resource to admit high bandwidth-consuming calls, such as video calls. Existing joint call admission control (JCAC) algorithms designed for heterogeneous wireless networks block/drop an incoming call when none of the available individual RATs in the network has enough bandwidth to admit the incoming call. Consequently, video calls experience high call blocking/dropping probability in the network. However, some calls such as multi-layer coded (scalable) video can be transmitted/ received over one or multiple RATs. This article proposes a JCAC algorithm that selects a single or multiple RATs for scalable video calls in heterogeneous wireless networks, depending on availability of radio resources in available RATs. Non scalable calls are always admitted into a single RAT by the algorithm. The aim of the proposed algorithm is to reduce call blocking/dropping probability for both scalable and non-scalable calls. An analytical model is developed for the proposed JCAC algorithm, and its performance is evaluated. Simulation results show that the proposed algorithm reduces call blocking/dropping probability in heterogeneous wireless networks.
\end{abstract}

Keywords: Heterogeneous wireless network, Joint radio resource management, Joint call admission control, Radio access technology, Markov chain, Mobile terminal

\section{Introduction}

There is an increasing demand for high bandwidthconsuming services such as real-time video and video streaming over wireless access networks [1,2]. Video calls generally consume high bandwidth in wireless networks. Consequently, video compression and bandwidth adaptation techniques have been used to reduce video call blocking/dropping probability in homogenous wireless networks, at the expense of the video quality at the receiver. The higher the video-compression ratio, the poorer the video quality at the receiver. Moreover, high frequency of bandwidth adaptation has also led to poor quality of service at the receiver.

However, multiple radio access technologies (RATs) are being deployed in the same geographical area, which

\footnotetext{
* Correspondence: bisi@crg.ee.uct.ac.za

Department of Electrical Engineering, University of Cape Town, Cape Town, South Africa
}

is referred to as heterogeneous wireless network environment. Consequently, the coexistence of different RATs in the same area has necessitated joint radio resource management (JRRM) for enhanced QoS provisioning and efficient radio resource utilization.

A multi-homed heterogeneous wireless network gives multimode terminals (MTs) the flexibility to be simultaneously connected to more than one RAT. Such simultaneous connections entails that packet stream of a scalable call will be split among multiple RATs in the heterogeneous wireless network. This flexibility can be exploited to improve connection-level QoS (call blocking/dropping probability) for high-bandwidth consuming applications such as multi-layer video.

A scalable video bit stream consists of one base layer and one or more enhancement layers, and therefore can provide better flexibility and efficiency [3]. By transmitting the layers of a scalable video over different RATs in

\section{实}


cooperative heterogeneous wireless networks, call blocking/dropping probability can be reduced in the networks. The foregoing is the focus of this article.

In cooperative heterogeneous wireless networks, a joint call admission control (JCAC) algorithm is used for making call admission decisions. A number of JCAC algorithms have been proposed for heterogeneous wireless networks, and a review of these JCAC algorithms given in [4]. However, these JCAC algorithms block/drop an incoming call when none of the available individual RATs in the heterogeneous network has enough bandwidth to support the call. Consequently, high bandwidth-demanding calls are easily blocked or dropped in the network, especially during the peak hours.

Sachs et al. [5] presented a framework for integrating different access systems into a multi-access system and for selecting the best suitable access network for users. They proposed a utility-based approach for evaluating different access allocation choices, which is based on user and network policies, the performance of access bearers, and the availability of access resources.

Hasib and Fapojuwo [6] presented an analysis of common radio resource management scheme for end-to-end QoS support in heterogeneous wireless networks.

Gelabert et al. [7] presented a Markovian approach to RAT selection in heterogeneous wireless networks. They developed an analytical model for RAT selection algorithms in a heterogeneous wireless network comprising GSM/EGDE and UMTS. The proposed algorithm selects just one RAT for each call.

Song and Zhuang [8] proposed a load-sharing call admission control scheme for voice and data services in an integrated cellular/WLAN network. In the proposed scheme, voice calls are preferably admitted to the cellular network, whereas data calls are admitted into both cellular and WLAN.

Falowo and Chan [9] proposed an adaptive JCAC and bandwidth management scheme for heterogeneous wireless networks with collocated cells. They developed an analytical model for the proposed scheme and evaluated its performance in terms of new call blocking probability (NCBP), handoff call dropping probability, and resource utilization.

In the JCAC schemes reviewed above, the possibility of splitting scalable calls and selecting multiple RATs for scalable calls in heterogeneous wireless networks were not considered. Therefore, this article focuses on the above topic.

The article proposes a JCAC scheme that reduces call blocking/dropping probability by selecting multiple RATs for an incoming call when none of the available single RATs has enough bandwidth to accommodate the incoming call. The JCAC algorithm is designed to simultaneously achieve the following objectives in heterogeneous wireless networks.

(1) Select a single RAT or multiple RATs for each call in the heterogeneous wireless.

(2) Degrade ongoing adaptive call(s) to the allowable minimum basic bandwidth unit (bbu), if necessary, to admit an incoming call.

(3) Reduce call blocking/dropping probability through multiple RAT selection.

(4) Prioritize handoff calls over new calls, and

(5) Guarantee the QoS requirement of all admitted calls.

In wireless networks, dropping an ongoing call is more annoying to users than blocking a new call. Therefore, handoff calls are usually prioritized over new calls. The proposed JCAC algorithm prioritizes handoff calls over new calls by using different rejection thresholds for new and handoff calls.

The contributions of this article are twofold. First, a JCAC algorithm for reducing call blocking/dropping probability in heterogeneous wireless networks is proposed. Second, an analytical model is developed for the proposed scheme, and its performance is evaluated in terms of NCBP and handoff call dropping probability.

To the best of authors' knowledge, this is the first JCAC scheme using multiple RAT selection and scalable call splitting for reducing call blocking/dropping probability in heterogeneous wireless networks.

The rest of this article is organized as follows. Section 2 discusses transmission of multi-layer video in heterogeneous wireless networks. In Section 3, the proposed JCAC algorithm is described. Section 4 presents the system model for the scheme. A Markov model is developed for the JCAC algorithm in Section 5. In Section 6, the performance of the proposed JCAC algorithm is evaluated through numerical simulations.

\section{Transmission of multi-layer video in heterogeneous wireless networks}

Multi-layer coded video can be transmitted/received over one or more RATs in a multi-homed heterogeneous wireless network where radio resources are jointly managed to reduce call blocking dropping probability. In this article, calls are classified into I classes, where I is the number of call classes. The calls are also broadly classified as scalable and non-scalable calls. The proposed algorithm always selects a single RAT for non-scalable calls whereas scalable calls can be admitted into one or multiple RATs, depending on availability of radio resources in each of the available RATs. Figure 1 shows a scalable class- $i$ video call where a digital video input is coded into $\left(D_{i}+1\right)$ layers. Layer 0 is the base layer 


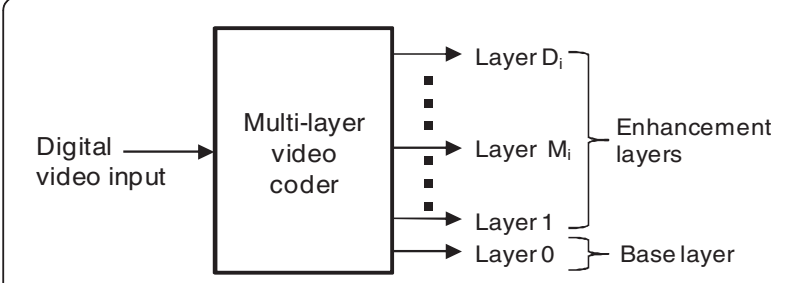

Figure 1 Illustration of layer-coded video.

whereas layers 1 to $D_{i}$ are enhancement layers where $D_{i}$ is the maximum number of enhancement layers, and $M_{i}$ is the minimum number of the enhancement layers required by the scalable class- $i$ calls.

As shown in Figure 1, a scalable class- $i$ call with minimum requested layers $\left(M_{i}+1\right)$ and maximum requested layers $\left(D_{i}+1\right)$ can be admitted into one or more RATs. For a scalable class- $i$ call, let $b_{i, 0}$ and $b_{i, d}$ denote the bandwidth requirements for the layer- 0 and layer- $d$, respectively, where $0<d \leq D_{i}$. The total minimum bandwidth requirement of a scalable class- $i$ call is given as

$$
b_{i, \min }=\sum_{x=0}^{M_{i}} b_{i, d}
$$

The total maximum bandwidth requirement of a scalable class- $i$ call is given as

$$
b_{i, \max }=\sum_{x=0}^{D_{i}} b_{i, d}
$$

For non-scalable, the bandwidth requirement is simply $b_{i, \min }=b_{i, \max }=b_{i,}$.

In a cooperative J-RAT heterogeneous wireless network, the requested bandwidth of an adaptive class- $i$ call, which varies between $b_{i, \min }$ and $b_{i, \max }$, can be allocated from a single RAT. However, if no single RAT has enough bbu to admit the incoming scalable call, with at least the minimum bandwidth requirement, $b_{i, \min }$, some ongoing scalable calls can be degraded (to the allowable minimum bbu) to free up some resources to accommodate the incoming call. However, if all scalable calls have be degraded to the allowable minimum, and yet, there is not a single RAT that has enough bbu to admit the incoming call (for example during the peak hour), the call is blocked of dropped.

To reduce blocking/dropping probability in heterogeneous wireless network, this article proposes a JCAC algorithm for transmission of multiple layers of scalable calls over multiple RATs, when it is not possible to admit the call, with requested minimum number of layers, over a single RAT.

For example, in a two-RAT heterogeneous wireless network where no single RAT has enough bbu to admit, a scalable class- $i$ call, requesting a minimum of $\left(1+M_{i}\right)$ layers, the layers can be split into two groups of layers (group- and group-2 layers): group-1 layers comprising layer- 0 to layer- $r_{i}$, and group-2 comprising layer- $\left(r_{i}+1\right)$ to layer- $M_{i}$, where $0 \leq r<M_{i}$. Then, layers in group-1 can be admitted into one RAT and layers in group two can be admitted into the other RAT provided that the two RATs support the scalable class- $i$ calls, and have enough radio resources to accommodate the group of layers. At the receiver, the two groups of layers are then combined.

Generally, in a J-RAT heterogeneous wireless networks, an $M_{i}$-layer class- $i$ call from a MT can be admitted into a single RAT or $T$ number of RATs, where $T \leq J$ and $T \leq\left(M_{i}+1\right)$ provided that up to T-RATs overlap and support the scalable class- $i$ call.

It is important to note that there are a number of challenges associated with transmission of multiple layers over two or more RATs in heterogeneous wireless networks. These challenges include synchronization of packets at the receiver, and combination of call different layers at the receiver. Buffering of packet stream of individual layer at the receiver before delivering them to the transport layer in the protocol stack can reduce some of the problems stated above. However, the above stated challenges are outside the scope of this article. The focus of this article is to develop a JCAC algorithm for selecting single or multiple RATs for incoming calls in heterogeneous wireless networks and investigates the effect of transmission of multi-layer video over multiple RATs on call blocking/dropping probability in heterogeneous wireless networks.

\section{Proposed JCAC for heterogeneous wireless networks}

The proposed JCAC scheme always selects a single RAT for non-scalable calls but selects a single or multiple RAT(s) for scalable calls, depending on availability of radio resources in the available RATs, and thereby reduces call blocking/dropping probability in heterogeneous wireless networks. Figure 2 illustrates the principle of multiple RAT selection and call layer splitting in a $J$-RAT heterogeneous wireless network, where $J$ is the number of RATs in the network.

As shown in Figure 2, an MT is requesting a scalable video-streaming session from a media server. None of the individual RATs in the J-RAT heterogeneous wireless networks has enough bbu to admit the incoming call (with the minimum bbu) because of the current load in each of the RATs. However, a combination of residual bbu in RAT 1 and RAT 3 will be sufficient to support the video streaming. Therefore, the proposed JCAC algorithm selects RAT 1 and RAT 3 for the call. The video layers are split between the two selected RATs and combined at the MT. The MT buffers the packet stream of individual group of layers for synchronization purposes 


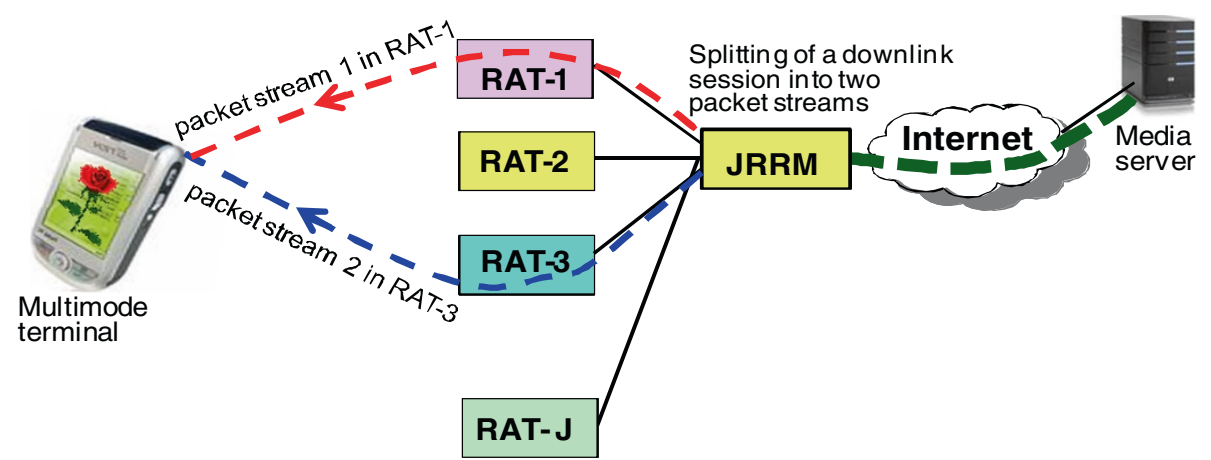

Figure 2 Multiple RAT selection in heterogeneous wireless network.

before delivering them to the transport layer in the protocol stack.

When a new call (session) is to be admitted, the proposed JCAC scheme decides the following: (1) whether the call can be admitted into the heterogeneous network or not, (2) whether some ongoing scalable call(s) should be degraded to accommodate the call or not, (3) whether the call should be split among multiple RATs or not (note that splitting is only allowed for scalable calls), and (4) what RAT(s) will be most suitable for admitting the incoming call. The JCAC scheme makes the above decisions based on the class of calls, bandwidth requirement of the call, and current load in each of the available RATs.

As shown in Figure 2, the JRRM module contains the JCAC algorithm. An MT wanting to make a call (initiate a session) sends a service request to the JRRM module. The JCAC algorithm in JRRM module then selects for the incoming call, a set of T RATs $(0 \leq T \leq \mathrm{J})$ from the available RATs in the heterogeneous network, where $T$ is the number of RATs selected. $T=0$ implies that the incoming call cannot be admitted into the heterogeneous network. Therefore, the call is blocked or dropped. $T=1$ implies that the incoming call can be admitted into just one of the available RATs. Hence, there is no layer splitting. $T>1$ implies that the incoming call is scalable and it will be admitted into more than one RAT.
Figure 3 shows the stages of the proposed JCAC algorithm. As shown in Figure 3, for a non-adaptive call, the proposed JCAC algorithm attempts to admit the call into a single RAT (Figure 3, 1(a)). If the call cannot be admitted into any of the available RATs because of insufficient bbu, the JCAC algorithm attempts to degrade some ongoing adaptive call(s) (to the allowable minimum bbu) in order to free some bbu for the incoming call (Figure 3, 2(a)). If there is no sufficient bbu to accommodate the incoming call in any of the available RATs after degrading the ongoing new calls to the allowable minimum bbu, the JCAC algorithm rejects the incoming call (Figure 3, 3(a)).

For an adaptive call, the proposed JCAC algorithm attempts to admit the call into a single RAT (Figure 3, 1(b)) with the requested bbu. If the call cannot be admitted into any of the available RATs because of insufficient bbu, the JCAC algorithm attempts to admit the adaptive call with the minimum bbu (Figure 3, 2(b)). If the call cannot be admitted into any of the available RATs because of insufficient bbu, the JCAC algorithm attempts to degrade some ongoing adaptive call(s) (to the allowable minimum bbu) in order to free some bbu for the incoming call (Figure 3, 3(b)). If the call cannot be admitted into any of the available RATs because of insufficient bbu, the JCAC algorithm attempts to admit the call

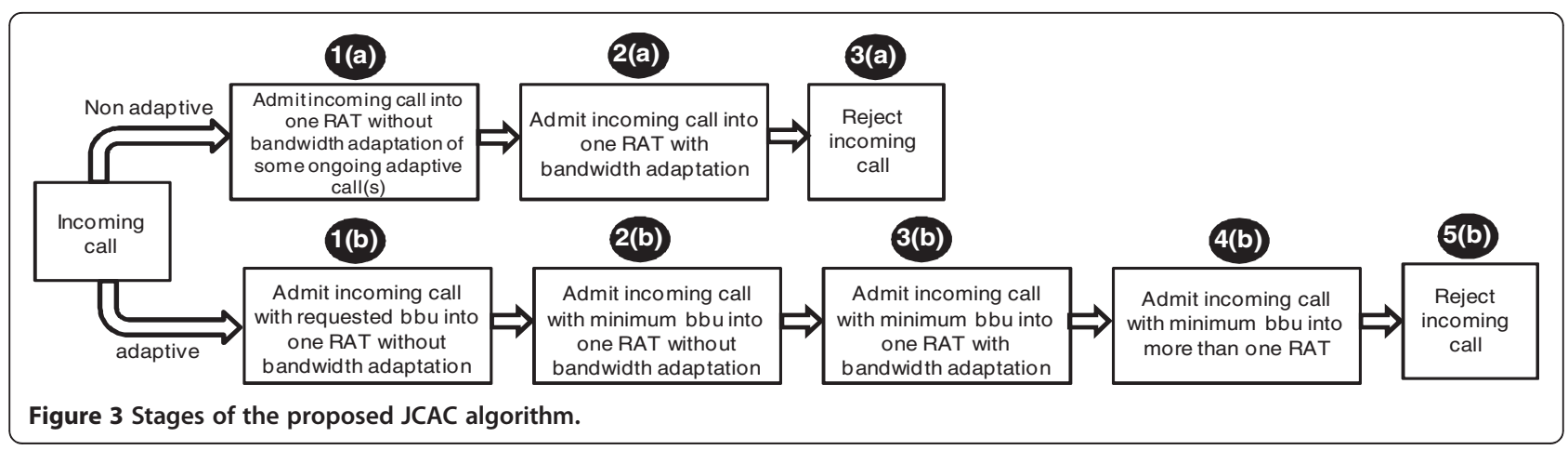




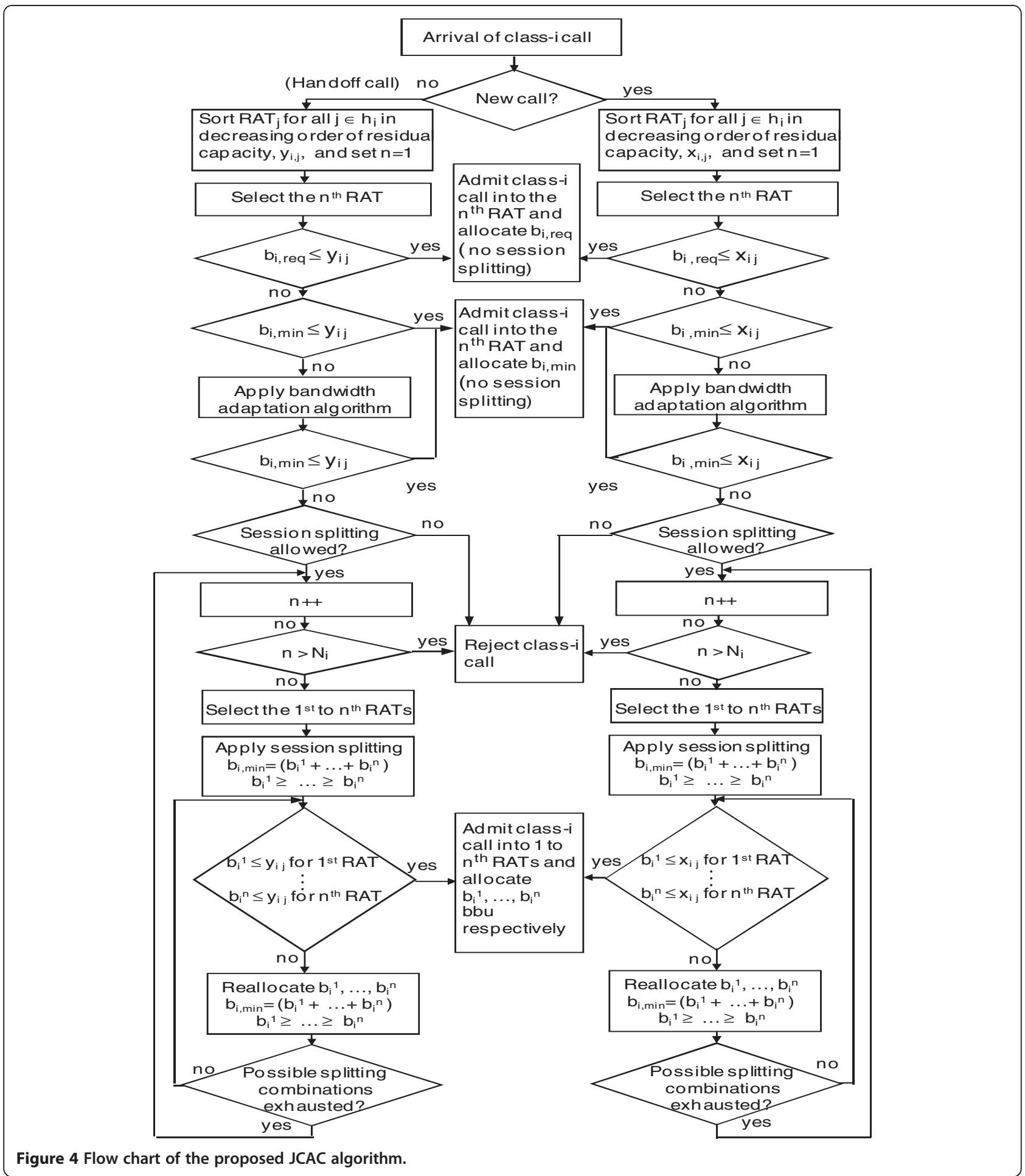

into more than one RAT (Figure 3, 4(b)). If there is no sufficient bbu to accommodate the incoming call in multiple RATs, the JCAC algorithm rejects the incoming call (Figure 3, 5(b)).

Figure 4 shows the proposed JCAC procedure, where, $x_{i, j}$ and $y_{i, j}$ denote the residual bandwidth available for new and handoff class- $i$ calls in RAT $j . h_{i, j}$ is the set of indices of RAT $j$ that support class- $i$ calls in the heterogeneous wireless networks. $N_{i}$ is the number of RATs that support class- $i$ calls in the heterogeneous wireless networks. $\quad b_{i, \text { req }}$ and $b_{i, \text { min }}$ are the total bandwidth requested and the minimum the bandwidth required by 


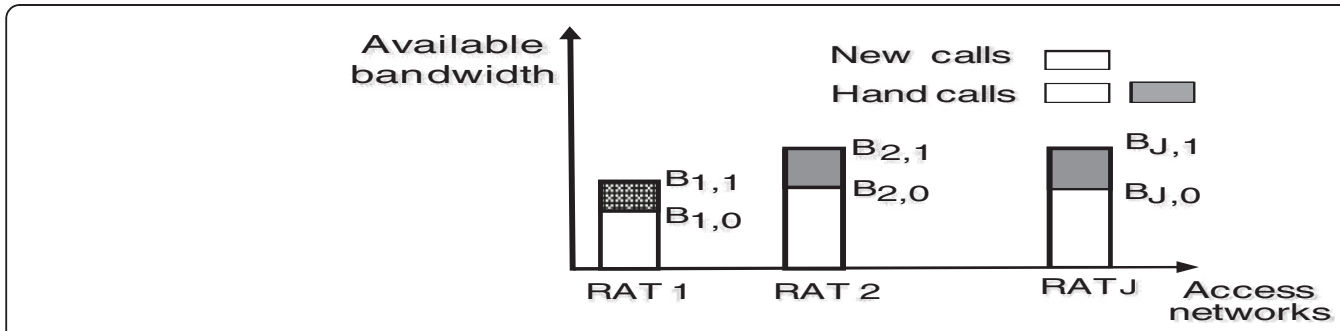

Figure 5 JCAC procedure.

class- $i$ call, respectively. $b_{i}^{j}$ is the fraction of bandwidth allocated to class $-i$ call in $\operatorname{RAT} j$, respectively.

In order to maintain lower handoff dropping probability over NCBP, different threshold are used for rejecting new and handoff calls. In RAT $j, B_{j, 1}$ and $B_{j, 0}$ are the threshold for rejecting handoff calls and new calls, respectively. Figure 5 shows the accessible bandwidth for new and handoff calls.

\section{System model and assumptions}

This article considers a heterogeneous wireless network, which consists of $J$ number of RATs with colocated cells, similar. Cellular networks such as GSM, GPRS, UMTS, EV-DO, LTE, etc. can have the same and fully overlapped coverage, which is technically feasible, and may also save installation cost $[10,11]$. Figures 6 and 7 illustrate a two-RAT heterogeneous cellular network. Figure 6, adapted from [12], is a typical heterogeneous cellular network comprising $3 \mathrm{G}$ WCDMA and LTE OFDMA. Figure 6 shows the co-located cells of the two-RAT heterogeneous wireless networks.

In heterogeneous cellular networks, radio resources can independently be or jointly managed. A situation where radio resources are jointly managed in the heterogeneous network and each cell in $\operatorname{RAT} j(j=1, \ldots, f)$ has a total of $B_{j, 0}$ bbu is considered. The physical meaning of a unit of radio resources (such as time slots, code sequence, etc.) is dependent on the specific technological implementation of the radio interface. However, no matter which multiple access technology (FDMA, TDMA, CDMA, or OFDMA) is used, system capacity could be interpreted in terms of effective or equivalent bandwidth. Therefore, in this article, bandwidth required by a call is denoted by bbu, which is similar to the approach used for wireless networks in [13].

Our approach is based on decomposing a heterogeneous cellular network into groups of co-located cells. As shown in Figure 7, cells 1a and 2a form a group of co-located cells. Similarly, cells $1 b$ and $2 b$ form another group of co-located cells, and so on.

A newly arriving call will be admitted into one or multiple cells in the group of co-located cells where the call is located. For example, in the two-RAT heterogeneous wireless network shown in Figure 7, an incoming call from an MT can be admitted into either of the two RATs (cell $1 \mathrm{~b}$ or cell $2 \mathrm{~b}$ ) in the group of collocated cells. Alternatively, the call can be admitted into both RATs (cells $1 \mathrm{~b}$ and $2 \mathrm{~b}$ ), with session splitting. Otherwise the call is blocked.

The correlation between the groups of co-located cells results from handoff connections between the cells of

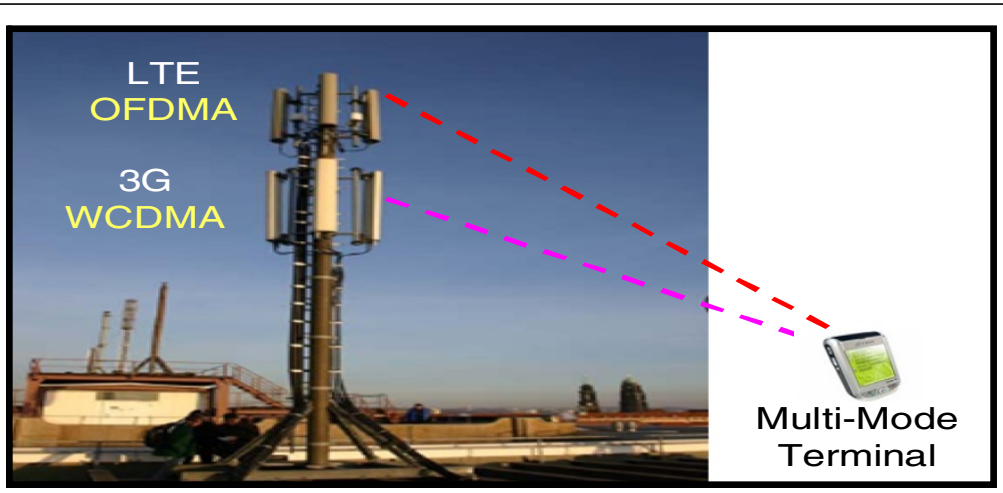

Figure 6 A typical two-RAT heterogeneous cellular network with co-located cells. 


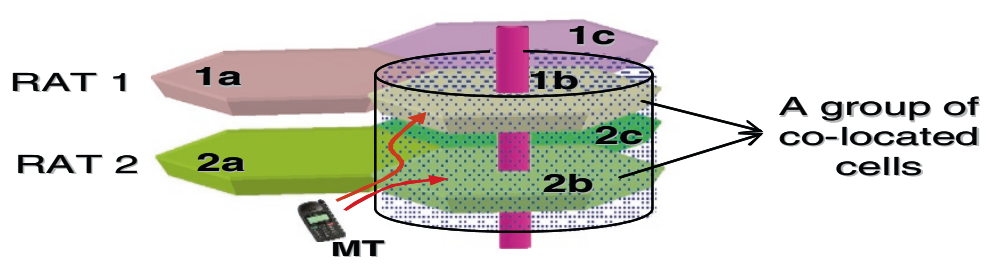

Figure 7 Co-located cells of a two-RAT heterogeneous cellular network.

corresponding groups. Under this formulation, each group of co-located cells can be modeled and analyzed individually. Therefore, this article focuses attention on a single group of co-located cells.

The heterogeneous network supports $I$ classes of calls. Each class- $i$ call (which can be scalable or non-scalable) requires a discrete bandwidth value, $b_{i}$. Each class is characterized by bandwidth requirements, arrival distribution, and channel holding time. Some classes of calls (e.g., video streaming) may support session splitting whereas some other classes of call (e.g., voice) may not support or require session splitting.

Following the general assumption in cellular networks, new and handoff class- $i$ calls arrive in the group of colocated cells according to Poisson process with rate $\lambda_{i}^{n}$ and $\lambda_{i}^{h}$, respectively [14]. Note that the arrival rates of a split Poisson process are also Poisson [15].

The channel holding time for class- $i$ calls is exponentially distributed with mean $1 / \mu i[14]$. Note that this set of assumptions has been used for heterogeneous wireless networks in the literature, and is found to be generally applicable in the environment where the number of mobile users is larger than the number of channels $[13,14]$.

\section{Markov model}

The JCAC policy described in Section 3 can be modeled as a multi-dimensional Markov chain. The state space of the group of co-located cells can be represented by a $\left(2 * I^{*} J^{*} K\right)$-dimensional vector given as

$$
\begin{aligned}
& \Omega=\left(m_{i, j, k}, n_{i, j, k}: i=1, \ldots, I, \quad j=1, \ldots, J\right. \\
& k=1, \ldots, K)
\end{aligned}
$$

The non-negative integer $m_{i, j, k}$ denotes the number of ongoing new class- $i$ calls (or sub-streams of class- $i$ calls) allocated $k$ bbu in RAT $j$, and the non-negative integer $n_{i, j, k}$ denotes the number of ongoing handoff class- $i$ calls (or sub streams of handoff class- $i$ calls) allocated $k$ bbu in $\operatorname{RAT} j$. Let $S$ denote the state space of all admissible states of the group of co-located cells as it evolves over time. An admissible state $s$ is a combination of the numbers of users in each class that can be supported simultaneously in the group of co-located cells while maintaining adequate QoS and meeting resource constraints. $k(1 \leq k \leq K)$ is an integer and it is the number of bbu allocated to call or substream of a call in a particular RAT. $K$ is the maximum number of bbu that can be allocated to any class- $i$ call (i.e., without session splitting).

\section{The state $S$ of all admissible states in the group of co-located cells is given as}

$$
\begin{aligned}
& S=\left\{\Omega=\left(m_{i, j, k}, n_{i, j, k}: i=1, \ldots, I, \quad j=1, \ldots, J,\right.\right. \\
& \left.k=1, \ldots, b_{i}\right): \sum_{i=1}^{I} \sum_{k=1}^{K} m_{i, j, k} \cdot k \leq B_{j, 0} \forall j \wedge, \\
& \left.\sum_{i=1}^{I} \sum_{k=1}^{K} m_{i, j, k} \cdot k+\sum_{i=1}^{I} \sum_{k=1}^{K} n_{i, j, k} \cdot k \leq B_{j, 1} \forall j\right\}
\end{aligned}
$$

Joint call admission decisions are taken in the arrival epoch. Every time a new or handoff class- $i$ call arrives in the group of co-located cells, the JCAC algorithm decides whether or not to admit the call, and in which set of RAT(s) to admit it. Note that a call admission decision is made only at the arrival of a call, and no call admission decision is made in the group of co-located cells when a call departs. When the system is in state $s$, an accept/ reject decision must be made for each type of possible arrival, i.e., an arrival of a new class- $i$ call, or the arrival of a handoff class- $i$ call in the group of co-located cells. For an incoming class- $i$ call, the following are the possible JCAC decisions in the arrival epoch.

(1) Reject the class- $i$ call (new or handoff) in the group of collocated cells, in which case the state $s$ does not evolve.

(2) Admit the class- $i$ call into only one RATs (no session splitting) in which case the state $s$ evolves.

(3) Admit the class- $i$ call into a set of RATs (with session splitting) in which case the state $s$ evolves. 
Thus, the call admission action space $A$ can be expressed as follows:

$$
\begin{aligned}
A= & \left\{a=\left(a_{1}^{n}, \ldots, a_{I}^{n}, a_{1}^{h}, \ldots, a_{I}^{h}\right):\right. \\
& a_{i}^{n}, a_{i}^{h} \in\left\{A_{0}, A_{1}, A_{2}, \ldots, A_{J}\right\} \\
A_{0}= & \{0\}, \text { number of elements in } A_{0}, \\
& n\left(A_{0}\right)={ }^{J} C_{0}=1 \\
A_{1}= & \{1,2, \ldots, J\}, n\left(A_{1}\right)={ }^{J} C_{1}=\mathrm{J} \\
A_{2}= & \{(1 \& 2), \ldots,(1 \& \mathrm{~J}),(2 \& 3), \ldots,(2 \& \mathrm{~J}), \ldots, \\
& ((J-1) \& \mathrm{~J})\}, n\left(A_{2}\right)={ }^{J} C_{2} \\
A_{J}= & \{(1 \& 2 \& \ldots \&(\mathrm{~J}-1) \& \mathrm{~J})\}, \mathrm{n}\left(\mathrm{A}_{\mathrm{J}}\right)={ }^{\mathrm{J}} \mathrm{C}_{\mathrm{J}}=1
\end{aligned}
$$

where $\mathbf{a}_{i}^{\mathrm{n}}$ denotes the action taken on arrival of a new class- $i$ call within the group of co-located cells, and $\mathbf{a}_{i}^{\mathrm{h}}$ denotes the action taken on arrival of a handoff class- $i$ call from an adjacent group of co-located cells. $a_{i}^{n}$ (or $\left.a_{i}^{h}\right) \in A_{0}$ means no RAT is selected for an incoming new (or handoff) class- $i$ call, therefore, the new (or handoff) class- $i$ call is rejected in the heterogeneous wireless network. $a_{i}^{n}$ (or $\left.a_{i}^{h}\right) \in A_{1}$ means one RAT is selected for the call, therefore, there is no session splitting and the new (or handoff) class- $i$ call is accepted into the selected single RAT. $a_{i}^{n}\left(\right.$ or $\left.a_{i}^{h}\right) \in A_{2}$ means two RATs are selected for the call, therefore, there is session splitting and the new (or handoff) class- $i$ call is split into two substreams and admitted into the selected two RATs. $a_{i}^{n}\left(\right.$ or $\left.a_{i}^{h}\right) \in A_{j}$ means $j$ RATs are selected for the incoming call. Thus, there is session splitting and the new (or handoff) class- $i$ call is split into $j$ substreams and admitted into the selected $j$ RATs.

For example, in a 3-RAT heterogeneous wireless network, it follows that

$$
\begin{aligned}
& A_{0}=\{0\}, A_{1}=\{1,2,3\}, \\
& A_{2}=\{(1 \& 2),(1 \& 3),(2 \& 3)\}, A_{3}=\{(1 \& 2 \& 3)\}, \\
& a_{i}^{n}, a_{i}^{h} \in\{0,1,2,3,(1 \& 2),(1 \& 3),(2 \& 3),(1 \& 2 \& 3)\}
\end{aligned}
$$

where $a_{i}^{n}$ (or $\left.a_{i}^{h}\right)=0$ means reject the new (or handoff) class- $i$ call. $a_{i}^{n}$ (or $\left.a_{i}^{h}\right)=1$ means accept the new (or handoff) class- $i$ call into RAT-1. $a_{i}^{n}$ (or $\left.a_{i}^{h}\right)=(1 \& 2)$ means split the scalable call session into two substreams and accept the new (or handoff) class- $i$ call subsreams into RAT-1 and RAT-2. $a_{i}^{n}$ (or $\left.a_{i}^{h}\right)=(1 \& 2 \& 3)$ means split the call session into three substreams and accept the new (or handoff) class- $i$ call subsreams into RAT-1, RAT-2, and RAT-3.
Based on its Markovian property, the JCAC algorithm can be model as a $\left.\left(2 * I^{*}\right)^{*} K\right)$-dimensional Markov chain. Let $\rho_{\text {new }_{i, j, k}}$ and $\rho_{\text {hani } i, k}$ denote the load generated by new class $-i$ calls and handoff class- $i$ calls, respectively, in RAT $-j$. Let $1 / \mu_{i}^{n}$ and $1 / \mu_{i}^{h}$ denote the channel holding time of new class- $i$ call and handoff class- $i$ call, respectively, and let $\lambda_{i, j, k}^{n}$ and $\lambda_{i, j, k}^{h}$ denote the arrival rates of new class- $i$ call (or sub-stream of new class- $i$ call) and handoff class- $i$ call (or sub-stream of handoff class- $i$ call) allocated $k$ bbu in RAT $j$, respectively, then

$$
\rho_{\text {new }}=\frac{\lambda_{i, j}^{n}}{\mu_{i}^{n}} \forall i, j, k
$$

$$
\rho_{\text {han } i j}=\frac{\lambda_{i, j, k}^{h}}{\mu_{i}^{h}} \forall i, j, k
$$

From the steady-state solution of the Markov model, performance measures of interest can be determined by summing up appropriate state probabilities. Let $P(s)$ denotes the steady-state probability that system is in state $\boldsymbol{s}(\boldsymbol{s} \in \boldsymbol{S})$. From the detailed balance equation, $P(s)$ is obtained as

$$
P(s)=\frac{1}{G} \prod_{i=1}^{I} \prod_{j=1}^{J} \prod_{k=1}^{K} \frac{\left(\rho_{\text {new }_{i, j, k}}\right)^{m_{i, j, k}}}{m_{i, j, k} !} \frac{\left(\rho_{\text {han }_{i, j, k}}\right)^{n_{i, j, k}}}{n_{i, j, k} !} \forall s \in S
$$

where $G$ is a normalization constant given by

$$
G=\sum_{s \in S} \prod_{i=1}^{I} \prod_{j=1}^{J} \prod_{k=1}^{K} \frac{\left(\rho_{\text {new }_{i, j, k}}\right)^{m_{i, j, k}}}{m_{i, j, k} !} \frac{\left(\rho_{\text {han }_{i, j, k}}\right)^{n_{i, j, k}}}{n_{i, j, k} !}
$$

\section{NCBP}

A new class- $i$ call is blocked in the group of co-located cells if the selected RAT(s) do not have enough bbu to accommodate the new call. Let $S_{b i} \subset S$ denote the set of states in which a new class- $i$ call is blocked in the group of collocated cells. It follows that 


$$
\begin{aligned}
& \begin{array}{c}
S_{b i}=\{s \in S \\
\left(b_{i, \min }+\sum_{i=1}^{I} \sum_{k=1}^{K} m_{i, r^{1,1}, k} \cdot k>B_{r^{1,1}, 0}, \vee\right.
\end{array} \\
& \left.b_{i, \min }+\sum_{i=1}^{I} \sum_{k=1}^{K}\left(m_{i, r^{1,1}, k}+n_{i, r^{1,1}, k}\right) \cdot k>B_{r^{1,1}, 1}\right) \forall r^{1,1} \in h_{i} \\
& \left(\alpha_{i}^{2,1, c} b_{i, \min }+\sum_{i=1}^{I} \sum_{k=1}^{\Lambda} m_{i, r^{2,1, k}} \cdot k>B_{r^{2,1}, 0} \vee\right. \\
& \left.\alpha_{i}^{2,1, c} b_{i, \min }+\sum_{i=1}^{I} \sum_{k=1}^{K}\left(m_{i, r^{2,1}, k}+n_{i, r^{2,1,}, k}\right) \cdot k>B_{r^{2,1}, 1}\right) \vee \\
& \left(\alpha_{i}^{2,2, c} b_{i, \min }+\sum_{i=1}^{I} \sum_{k=1}^{K} m_{i, r^{2,2}, k} \cdot k>B_{r^{2,2}, 0} \vee\right. \\
& \left.\alpha_{i}^{2,2, c} b_{i, \min }+\sum_{i=1}^{I} \sum_{k=1}^{K}\left(m_{i, r^{2,2,}, k}+n_{i, r^{2,2}, k}\right) \cdot k>B_{r^{2,2}, 1}\right) \\
& \forall\left(\left(r^{2,1} \neq r^{2,2}\right) \wedge\left(r^{2,1}, r^{2,2}\right) \in h_{i}\right) \\
& \left.\forall c=\left[1,2, \ldots, c_{2}\right]\right) \\
& \text {. } \\
& \left(\alpha_{i}^{N_{i}, 1, c} b_{i, \min }+\sum_{i=1}^{I} \sum_{k=1}^{\stackrel{\bullet}{K}} m_{i, r^{N_{i},}, k} \cdot k>B_{r^{N_{i}, 1}, 0} \vee\right. \\
& \left.\alpha_{i}^{N_{i}, 1, c} b_{i, \min }+\sum_{i=1}^{I} \sum_{k=1}^{K}\left(m_{i, r^{N_{i}, 1}, k}+n_{i, r^{N_{i}, 1}, k}\right) \cdot k>B_{r^{N_{i}, 1}, 1}\right) \\
& \left(\alpha_{i}^{N_{i}, N_{i}, c} b_{i, \min }+\sum_{i=1}^{I} \sum_{k=1}^{K} m_{i, r^{N}, N_{i}, k} \cdot k>B_{r^{N} N_{i}, N_{i}, 0} \vee\right. \\
& \left.\alpha_{i}^{N_{i}, N_{i}, c} b_{i, \min }+\sum_{i=1}^{I} \sum_{k=1}^{K}\left(m_{i, r_{i}, N_{i}, k}+n_{i, r^{N_{i}, N_{i}, k}}\right) \cdot k>B_{r N_{i}, N_{i}, 1}\right) \\
& \forall\left(\left(r^{N_{i}, 1} \neq \ldots \neq r^{N_{i}, N_{i}}\right) \wedge\left(r^{N_{i}, 1}, \ldots, r^{N_{i}, N_{\mathrm{i}}}\right) \in h_{i}\right) \\
& \left.\left.\forall c=\left[1,2, \ldots, c_{n}\right]\right)\right\}
\end{aligned}
$$

where $N_{i}$ is the number of RATs that support class- $i$ calls and $r^{p, q}$ is the indices of $q$ th RAT of the selected $p$ RATs for an incoming class- $i$ call. $\alpha_{i}^{p, q, c}$ is the fraction of bbu allocated to an incoming class $-i$ call in the $q$ th RAT of the selected $p$ RATs with session splitting combination $c$.

Thus, the NCBP, $P_{b_{i}}$, for a class- $i$ call in the group of co-located cells is given by

$$
P_{b_{i}}=\sum_{s \in S_{b_{i}}} P(s)
$$

\section{Handoff call dropping probability}

A handoff class- $i$ call is dropped in the group of colocated cells if the selected RAT(s) do not have enough bbu to accommodate the handoff call. Let $S_{d_{i}} \subset S$ denote the set of states in which a handoff class- $i$ call is dropped in the group of co-located cells.

$$
\begin{aligned}
& S_{d i}=\{s \in \mathrm{S}: \\
& \left(b_{i, \min }+\sum_{i=1}^{I} \sum_{k=1}^{K}\left(m_{i, r^{1,1}, k}+n_{i, r^{1,1}, k}\right) \cdot k>B_{r^{1,1}, 1}\right) \forall r^{1,1} \in h_{i} \\
& \left(\alpha_{i}^{2,1, c} b_{i, \min }+\sum_{i=1}^{I} \sum_{k=1}^{K}\left(m_{i, r^{2,1}, k}+n_{i, r^{2,1}, k}\right) \cdot k>B_{r^{2,1}, 1}\right) \vee \\
& \left(\alpha_{i}^{2,2, c} b_{i, \min }+\sum_{i=1}^{I} \sum_{k=1}^{K}\left(m_{i, r^{2,2, k}}+n_{i, r^{2,2,}, k}\right) \cdot k>B_{r^{2,2,1}, 1}\right) \\
& \forall\left(\left(r^{2,1} \neq r^{2,2}\right) \wedge\left(r^{2,1}, r^{2,2}\right) \in h_{i}\right) \\
& \left.\forall c=\left[1,2, \ldots, c_{2}\right]\right) \\
& \wedge \\
& \left(\left(\alpha_{i}^{N_{i}, 1, c} b_{i, \min }+\sum_{i=1}^{I} \sum_{k=1}^{K}\left(m_{i, r_{i}, 1, k}+n_{i, r^{N_{i}, 1}, k}\right) \cdot k>B_{r^{N_{i}, 1}, 1}\right)\right. \\
& \begin{array}{c}
\vee \\
\vdots \\
\\
\vee
\end{array} \\
& \left(\alpha_{i}^{N_{i}, N_{i}, c} b_{i, \min }+\sum_{i=1}^{I} \sum_{k=1}^{K}\left(m_{i, r_{i}, N_{i}, k}+n_{i, r^{N_{i}, N_{i}, k}}\right) \cdot k>B_{r^{N_{i}, N_{i}, 1}}\right) \\
& \forall\left(\left(r^{N_{i}, 1} \neq \cdots \neq r^{N_{i}, N_{i}}\right) \wedge\left(r^{N_{i}, 1}, \ldots r^{N_{\mathrm{i}}, N_{\mathrm{i}}}\right) \in h_{\mathrm{i}}\right) \\
& \left.\left.\forall c=\left[1,2, \ldots, c_{N i}\right]\right)\right\}
\end{aligned}
$$

Thus, the handoff class- $i$ call dropping probability (HCDP) for a class- $i$ call, $P_{d_{i}}$, in the group of co-located cells is given by

$$
P_{d_{i}}=\sum_{s \in S_{d_{i}}} P(s)
$$

\section{Simulation results}

In this section, the performance of the proposed JCAC scheme is evaluated via numerical simulation, using as an example of heterogeneous wireless network, a twoRAT heterogeneous cellular network supporting two classes of calls namely scalable video streaming calls (designated as class-1 calls) and voice calls (designated as class- 2 calls). In the example, class- 1 calls allows layer splitting whereas class-2 calls do not allow splitting. Class-1 calls can be admitted into a single RAT or split into two groups of layers, and then admitted into the two available RATs (i.e., $\left.a_{1}^{n}, a_{1}^{h} \in\{0,1,2,(1 \& 2)\}\right)$. Class2 calls can be admitted into a single RAT (i.e., $a_{2}^{n}, a_{2}^{h} \in$ $\{0,1,2\})$. The system parameters used are as follows: $B_{1,0}=0.8 B_{1,1}, B_{2,0}=0.8 B_{2,1}, b_{1, \text { req }}=b_{1, \text { min }}$ (the values are 
Table 1 Total bbu of class-1 calls and allowed splitting proportions

\begin{tabular}{lll}
\hline Scenario & $\boldsymbol{b}_{\mathbf{1 , \operatorname { m i n }}}$ & Splitting proportion \\
\hline $\mathbf{1}$ & $\mathbf{6}$ & $3: 3$ \\
$\mathbf{2}$ & $\mathbf{6}$ & $4: 2$ \\
$\mathbf{3}$ & $\mathbf{6}$ & $5: 1$ \\
$\mathbf{4}$ & $\mathbf{6}$ & No splitting \\
$\mathbf{5}$ & $\mathbf{8}$ & $4: 4$ \\
$\mathbf{6}$ & $\mathbf{8}$ & $5: 3$ \\
$\mathbf{7}$ & $\mathbf{8}$ & $6: 2$ \\
$\mathbf{8}$ & $\mathbf{8}$ & $7: 1$ \\
$\mathbf{9}$ & $\mathbf{8}$ & No splitting \\
\hline
\end{tabular}

shown in Table 1), $b_{2, \text { req }}=3 \mathrm{bbu}, \mu_{1}=0.5 \mu_{2}=0.5$. Table 1 shows the total bbu of class- 1 calls and splitting proportions allowed in each scenario. For example, in scenario 1 , total bbu required by class- 1 calls is 6 . If none of the two RATs has 6 bbu to admit an incoming class- 1 call. The 6 bbu required by the incoming call can be split into two equal proportions (3:3). The call can then be admitted into the two RATs, given that the remaining bbu in each of the RATs is up to 3 bbu. In scenario 4, the total bbu required by class- 1 calls is 6 ; session splitting is not allowed, and an incoming scalable call can only be admitted into a single RAT.

As shown in Table 1, nine scenarios are considered in the performance evaluation of the proposed JCAC scheme through numerical simulations conducted in MATLAB. For comparison purposes, the nine scenarios are grouped into two: scenarios 1-4 and 5-9.

\section{Effect of layer splitting on NCBP and HCDP (scenarios 1-4)}

Figures $8,9,10$, and 11 show the effect layer splitting on NCBP ( $\mathrm{Pb} 1$ and $\mathrm{Pb} 2$ ) and HCDP (Pd1 and $\mathrm{Pd} 2$ ) for class- 1 and class- 2 calls, when $b_{1}=6$ and $b_{2}=3$.

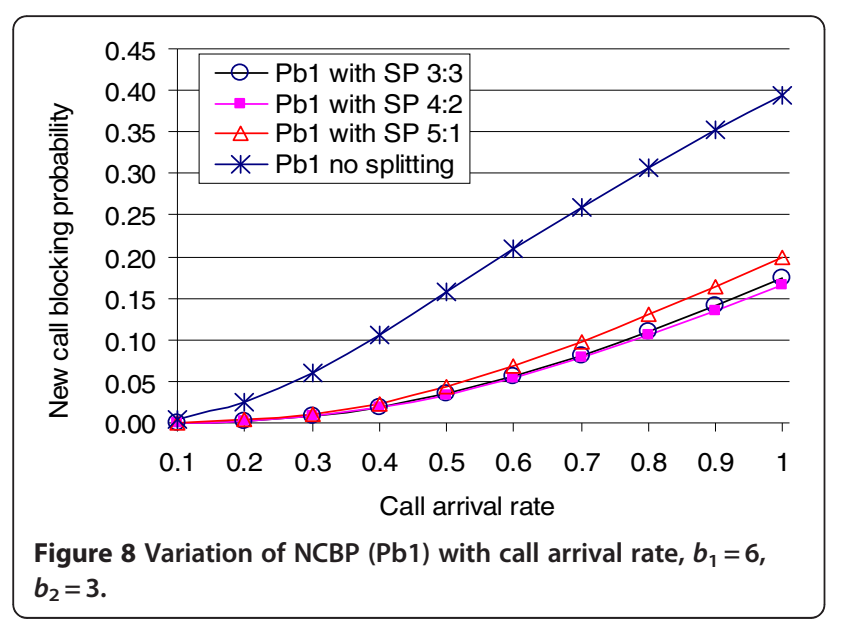

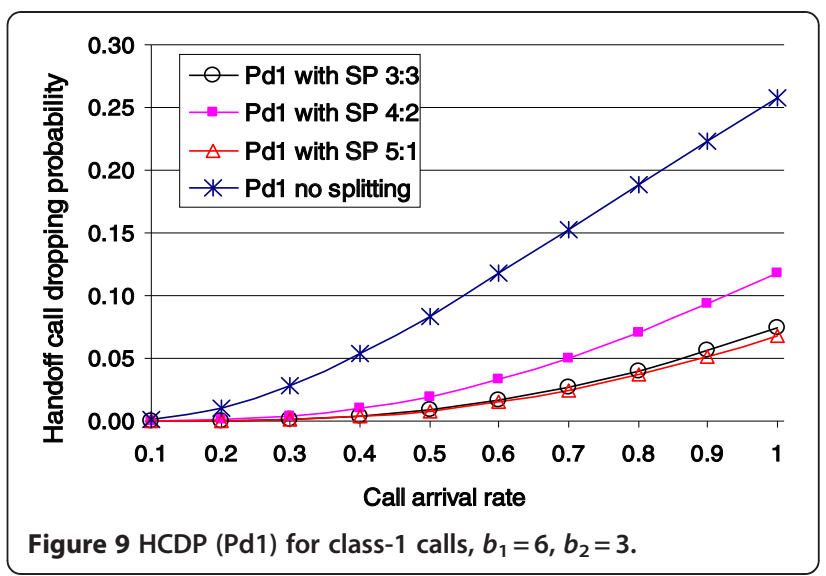

As showed in Figure 8, the NCBP (Pb1) increases with increase in call arrival rate for the four scenarios. However, the $\mathrm{Pb} 1$ of scenarios $1-3$ are much less than the $\mathrm{Pb} 1$ of scenario 4 . The reason is that in scenarios $1-3$, layer splitting is allowed for scalable calls. Therefore, if a new class- 1 call cannot be admitted into a single RAT, it can be split into two groups of layers, and then be admitted into the two RATs. Thus, layer splitting reduces NCBP in heterogeneous wireless networks.

Similarly, as shown in Figure 9, the HCDP (Pd1) increases with increase in call arrival rate for the four scenarios. However, the Pd1 of scenarios 1-3 are much less than the $\mathrm{Pb} 1$ of scenario 4 . The reason is that in scenarios $1-3$, layer splitting is allowed for scalable calls. Therefore, if a handoff class- 1 call cannot be admitted into a single RAT, it can be split into two groups of layers, and then be admitted into the two RATs. Thus, layer splitting reduces handoff call dropping probability in heterogeneous wireless networks.

Figure 10 shows the NCBP for class-2 calls. It can also be seen that NCBP (Pb2) increases with increase in call arrival rate for the four scenarios. However, the $\mathrm{Pb} 2$ of scenarios 1-3 are much less than the $\mathrm{Pb} 2$ of scenario 4. Note that session splitting is not allowed

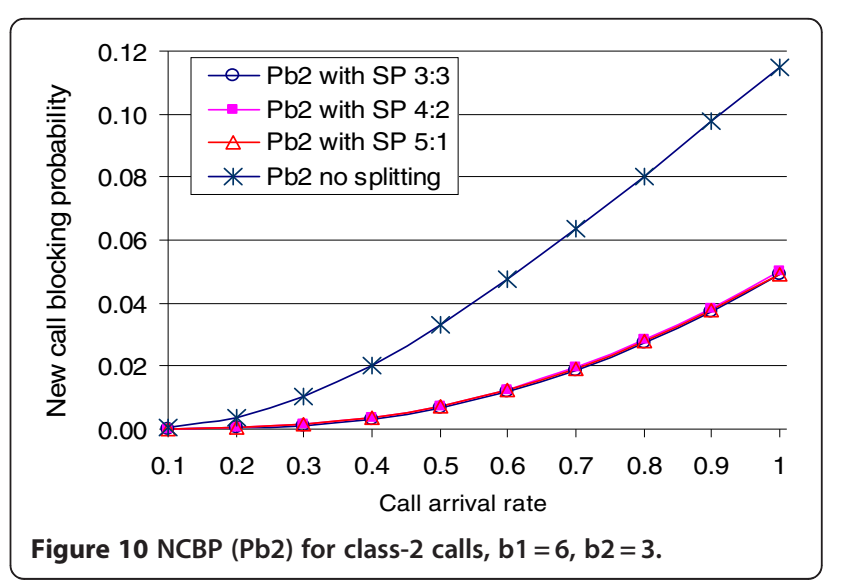




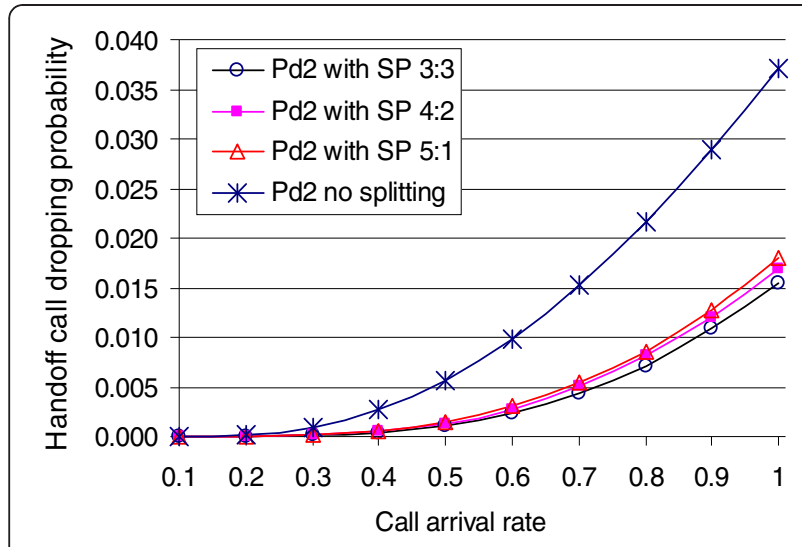

Figure $11 \mathrm{HCDP}(\mathrm{Pd} 2)$ for class- 1 calls, $b_{1}=6, b_{2}=3$.

for class two calls. The splitting proportions indicated in Figure 10 are applied to class-1 calls. However, they have positive effect on both new class-1 calls (Figure 8) and new class-2 calls (Figure 10). In other words, splitting of class- 1 calls in the heterogeneous wireless network reduces NCBP for both class-1 and class-2 calls.

Figure 11 shows the HCDP for class- 2 calls. It can also be seen that NCBP (Pd2) increases with increase in call arrival rate for the four scenarios. However, the Pd2 of scenarios 1-3 are much less than the $\mathrm{Pd} 2$ of scenario 4 . Splitting of class-1 calls reduces handoff call dropping probability for both handoff class-1 calls (Figure 9) and handoff calss-2 calls (Figure 11).

\section{Effect of layer splitting on NCBP and HCDP (scenarios 5-9)}

Figures 12, 13, 14, and 15 show the effect layer splitting on NCBP (Pb1 and Pb2) and HCDP (Pd1 and Pd2) for class- 1 and class- 2 calls, when $b_{1}=8$ and $b_{2}=3$.

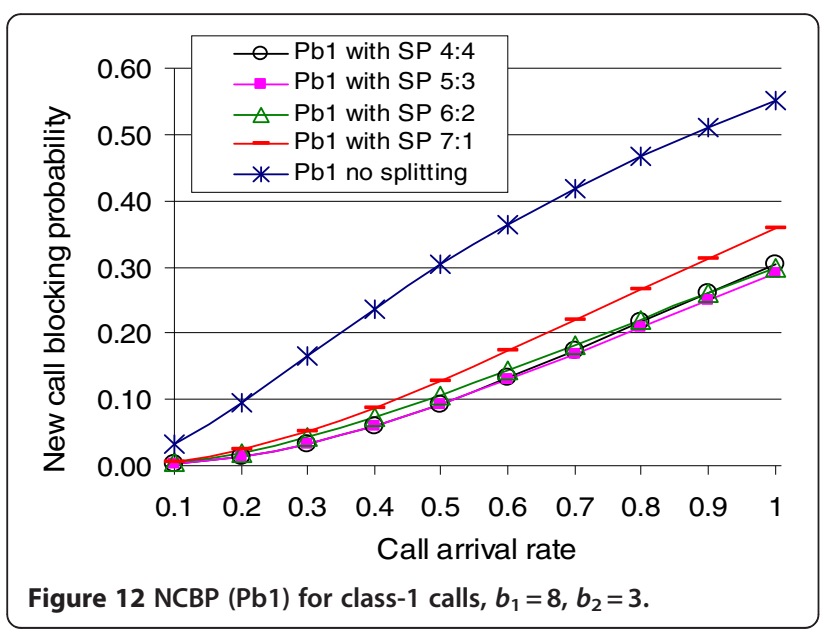

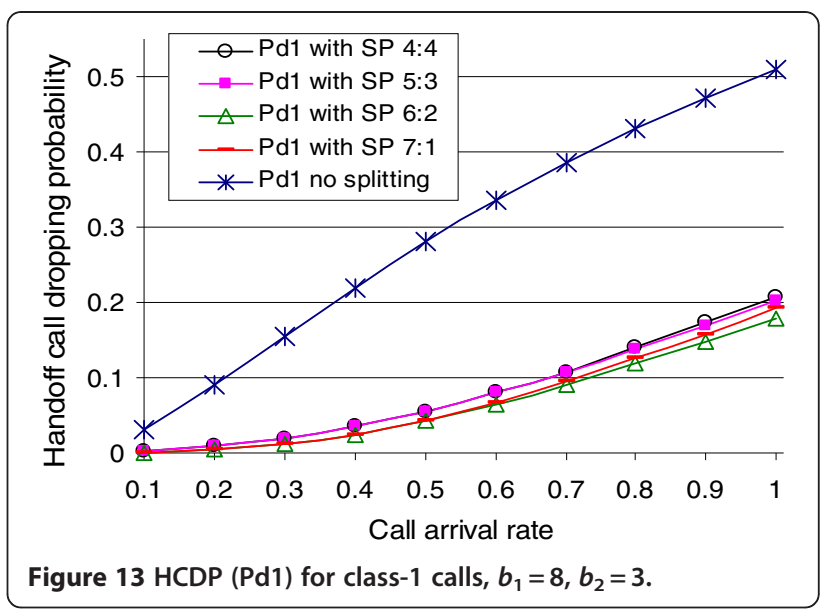

As showed in Figure 12, the NCBP (Pb1) increases with increase in call arrival rate for the four scenarios. However, the $\mathrm{Pb} 1$ of scenarios 5-8 are much less than the $\mathrm{Pb} 1$ of scenario 9. Thus, layer splitting reduces NCBP in heterogeneous wireless networks.

Similarly, as shown in Figure 13, the HCDP (Pd1) increases with increase in call arrival rate for the four scenarios. However, the Pd1 of scenarios 5-8 are much less than the Pd1 of scenario 9. Thus, layer splitting reduces handoff call dropping probability in heterogeneous wireless networks.

Figure 14 shows the NCBP for class-2 calls. It can also be seen that NCBP $(\mathrm{Pb} 2)$ increases with increase in call arrival rate for the four scenarios. However, the $\mathrm{Pb} 2$ of scenarios $5-8$ are much less than the $\mathrm{Pb} 2$ of scenario 9. Thus, splitting of class-1 calls reduces NCBP for both new class-1 (Figure 11) and class-2 calls (Figure 14).

Figure 15 shows the HCDP for class- 2 calls. It can also be seen that NCBP (Pd2) increases with increase in call arrival rate for the four scenarios. However, the Pd2 of scenarios 5-8 are much less than the Pd2 of scenario 9.

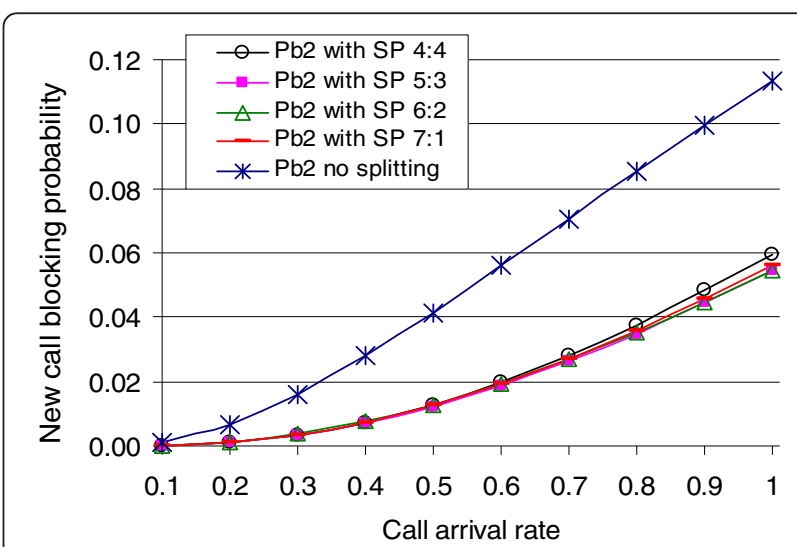

Figure $14 \mathrm{NCBP}(\mathrm{Pb} 2)$ for class- 1 calls, $b_{1}=8, b_{2}=3$. 


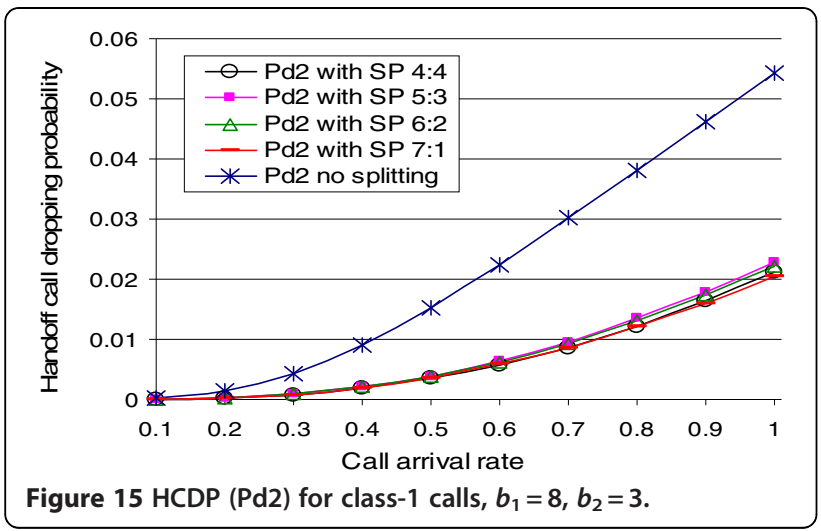

Thus, splitting of class-1 calls reduces handoff call dropping probability for both handoff class- 1 and class- 2 calls.

\section{Conclusion}

In this article, a JCAC scheme for reducing call blocking/dropping probability in cooperative heterogeneous wireless networks has been developed. The algorithm uses multiple RAT selection and splitting of scalable call to reduce call blocking/dropping probability in heterogeneous wireless networks. An analytical model has been developed for the proposed JCAC scheme and two performance metrics namely call blocking probability and call dropping probability have been derived. Performance of the proposed JCAC scheme is evaluated and compared with that of a JCAC scheme that does not support layer splitting. Simulation results show that the proposed JCAC scheme reduces call blocking/dropping probability for both scalable and non-scalable calls in heterogeneous wireless networks.

\section{Competing interests}

The authors declare that they have no competing interests.

Received: 17 June 2011 Accepted: 18 May 2012

Published: 17 July 2012

\section{References}

1. E Maani, Y Luo, P Pahalawatta, A Katsaggelos, Packet scheduling for scalable video streaming over lossy packet access networks, in Proceedings of 16th International Conference on Computer Communications and Networks, Turtle Bay Resort (16th International Conference on Computer Communications and Networks, Honolulu, Hawaii, USA, 2007), pp. 591-596

2. $\mathrm{P} L \mathrm{Li}, \mathrm{H}$ Zhang, B Zhao, S Rangarajan, Fast optimal resource allocation for scalable video multicast in broadband wireless networks. Proceedings of the IEEE International Conference on Communications, 1-6 (2010). Cape Town, South Africa

3. Z Chen, M Li, Y Tan, Perception-aware multiple scalable video streaming over WLANs. IEEE Signal Processing Letters 17(7), 675-678 (2010)

4. OE Falowo, HA Chan, Joint call admission control algorithms: requirements, approaches, and design considerations. Elsevier J.: Computer. Communications 31(6), 1200-1217 (2008). doi:10.1016/j. comcom.2008.10.044. 2008

5. J Sachs, M Prytz, I Gebert, Multi-access management in heterogeneous networks. Int. J. Wirel. Personal Communications 48(1), 7-32 (2009). January
6. A Hasib, AO Fapojuwo, Analysis of common radio resource management scheme for end-to-end QoS support in multiservice heterogeneous wireless networks. IEEE Trans. Veh. Technology 57(4), 2426-2439 (2008)

7. X Gelabert, J Perez-Romero, O Sallent, R Agustı, A Markovian approach to radio access technology selection in heterogeneous multiaccess/ multiservice wireless networks. IEEE Trans. Mob. Computing 7(10), 1257-1270 (2008). October

8. W Song, W Zhuang, Multi-service load sharing for resource management in the cellular/WLAN integrated network. IEEE Trans. Wireless Communication 8(2), 725-735 (February 2009)

9. OE Falowo, HA Chan, Adaptive bandwidth management and joint call admission control to enhance system utilization and QoS in heterogeneous wireless networks. EURASIP J. Wireless Communications and Networking (11) (2007). doi:10.1155/2007/34378. July 2007

10. W Zhang, Performance of real-time and data traffic in heterogeneous overlay wireless networks, in Proceedings of the 19th International Teletraffic Congress (ITC 19), Beijing, 2005, ed. by X.J. Liang, Z.H. Xin, V.B. Iversen, G.S. Kuo (Beijing University of Posts and Telecommunications Press, Beijing, China, 2005), pp. 859-868

11. H Holma, A. Toskala, WCDMA for UMTS, 2nd edn. (John Wiley \& Sons, New York, 2001)

12. G Fettweis, Current frontiers in wireless communications: fast \& green \& dirty, keynote presentation, in IEEE Wireless Communications \& Networking Conference (WCNC) (, Budapest, Hungary, 2009). 5-8 April 2009; http://www. ieee-wcnc.org/2009/downloads/Gerhard.pdf

13. N Nasser, H Hassanein, Dynamic threshold-based call admission framework for prioritized multimedia traffic in wireless cellular networks. Proceedings of the IEEE Global Telecommunications Conference (GLOBECOM 2004) 2, 644-649 (2004)

14. W Ni, W Li, M Alam, Determination of optimal call admission control policy in wireless networks. IEEE Trans. Wirel. Commun. 8(2), 1038-1044 (2009)

15. DP Bertsekas, JN Tsitsiklis, Introduction to Probability (Athena Scientific (Belmont, MA, 2002)

\section{doi:10.1186/1687-1499-2012-221}

Cite this article as: Falowo and Chan: Multiple-RAT selection for reducing call blocking/dropping probability in cooperative heterogeneous wireless networks. EURASIP Journal on Wireless Communications and Networking 2012 2012:221.

\section{Submit your manuscript to a SpringerOpen ${ }^{\mathcal{D}}$ journal and benefit from:}

- Convenient online submission

- Rigorous peer review

- Immediate publication on acceptance

- Open access: articles freely available online

- High visibility within the field

- Retaining the copyright to your article

Submit your next manuscript at $>$ springeropen.com 